\title{
Universal geometric coefficients for the four-punctured sphere (Extended Abstract)
}

\author{
Emily Barnard|; Emily Meehan "s Shira Polster|" and Nathan Reading \\ Department of Mathematics, North Carolina State University, Raleigh, NC, USA
}

\begin{abstract}
We construct universal geometric coefficients for the cluster algebra associated to the four-punctured sphere and obtain, as a by-product, the g-vectors of cluster variables. We also construct the rational part of the mutation fan. These constructions rely on a classification of the allowable curves (the curves which can appear in quasi-laminations). The classification allows us to prove the Null Tangle Property for the four-punctured sphere, thus adding this surface to a short list of surfaces for which this property is known. The Null Tangle Property then implies that the shear coordinates of allowable curves are the universal coefficients. We compute these shear coordinates to obtain universal geometric coefficients.

Résumé. Nous construisons des coefficients géométriques universels pour l'algèbre amassée associée à la sphère privée de 4 points, et obtenons ce faisant les g-vecteurs des variables d'amas. Nous construisons aussi la partie rationnelle de l'éventail de mutation. Ces constructions reposent sur la classification des courbes admissibles (les courbes qui peuvent apparaître dans les quasi-laminations). Cette classification nous permet de prouver la "Null Tangle Property" pour la sphère privée de 4 points, ajoutant ainsi cette surface à la courte liste de surfaces pour lesquelles cette propriété est connue. La "Null Tangle Property" implique alors que les coordonnées de décalage des courbes admissibles sont les coefficients universels. Nous calculons ces coordonnées de décalage pour obtenir les coefficients géométriques universels.
\end{abstract}

Keywords: cluster algebra, four-punctured sphere, Null Tangle Property, universal geometric coefficients

\section{Introduction}

The fundamental combinatorial datum specifying a cluster algebra is an exchange matrix, an $n \times n$ skew-symmetrizable (and in the special case considered here, skew-symmetric) integer matrix $B$. The remaining data specifying a cluster algebra can be thought of as a choice of coefficients. We consider the broad class of cluster algebras of geometric type, in which the choice of coefficients is a collection of vectors (geometric coefficients), each with $n$ entries. The usual definition of cluster algebras of geometric type is [4, Definition 2.12], but we work with the broader definition given in [7, Definition 2.8]. The relationship between the two definitions is described in [7, Remark 2.9].

\footnotetext{
${ }^{\dagger}$ Email: esbarnarencsu. edu. Supported in part by NSF grants DMS-0943855 and CCF-1017217.

‡Email: emeehan encsu . edu. Supported in part by NSF grants DMS-0943855 and CCF-1017217.

Email: spolsteencsu.edu. Supported in part by NSF grants DMS-0943855 and CCF-1017217.

TEmail: reading@math. ncsu . edu. Supported in part by NSF grant DMS-1101568.
} 


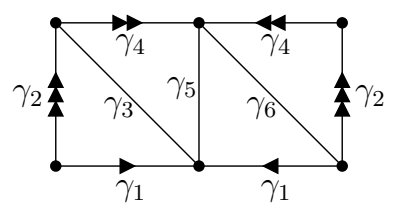

$$
\left[\begin{array}{rrrrrr}
0 & 1 & -1 & 0 & 1 & -1 \\
-1 & 0 & 1 & -1 & 0 & 1 \\
1 & -1 & 0 & 1 & -1 & 0 \\
0 & 1 & -1 & 0 & 1 & -1 \\
-1 & 0 & 1 & -1 & 0 & 1 \\
1 & -1 & 0 & 1 & -1 & 0
\end{array}\right]
$$

Fig. 1: A triangulation of the 4-punctured sphere and its signed adjacency matrix

Universal geometric coefficients are a choice of coefficients that are universal in the sense that any other choice of geometric coefficients can be obtained from the universal geometric coefficients by coordinate specialization. Constructing geometric coefficients is equivalent to constructing a basis, in the sense of mutation-linear algebra with respect to $B$. That is, we find a collection of vectors that is independent in a very strong sense and spanning in a similar strong sense. Closely related to this notion of basis is the mutation fan $\mathcal{F}_{B}$, a complete fan that encodes the combinatorics and geometry of mutations of $B$. In particular, one can read off this basis from the rays of $\mathcal{F}_{B}$.

In [2, 3], it was shown that certain cluster algebras are modeled by the combinatorics and geometry/topology of triangulated surfaces. The goal of this paper is to construct universal geometric coefficients and describe the mutation fan for the exchange matrix $B$ encoded by a certain triangulation $T$ of a 2-sphere. The triangulation $T$, a combinatorial tetrahedron, is shown in Figure 1, along with its signed adjacency matrix $B=B(T)$. (See Definition 3.3.) Arrows in the picture indicate edgeidentifications, and the arcs defining edges of $T$ are labeled $\gamma_{i}$. We write $B$ with rows and columns indexed by $\gamma_{1}, \gamma_{2}, \gamma_{3}, \gamma_{4}, \gamma_{5}, \gamma_{6}$ in that order. The four vertices of the triangulation are called punctures, because cluster algebras associated to $B$ can be understood in terms of the geometry of the 4-punctured sphere, with $T$ taken to be an ideal triangulation. Universal coefficients for exchange matrices arising from triangulated surfaces are considered in general in [8].

Geometric coefficients are encoded by the interactions between two classes of curves, tagged arcs and allowable curves. Both classes are equipped with a notion of compatibility. Seeds in the cluster algebra correspond to tagged triangulations - maximal collections of pairwise compatible tagged arcs. One example is the triangulation shown in Figure 1 The intersections between an allowable curve $\lambda$ and the arcs in a tagged triangulation $T$ are encoded in a vector $\mathbf{b}(T, \lambda)$ called the shear coordinates or shear coordinate vector of $\lambda$ with respect to $T$. The rational lamination fan $\mathcal{F}_{\mathbb{Q}}(T)$ is the fan whose cones are the nonnegative spans of the shear coordinate vectors of collections of pairwise compatible allowable curves with respect to $T$. Assuming a property of the surface called the Null Tangle Property, universal coefficients are the shear coordinates of allowable curves and the rational cones in the mutation fan $\mathcal{F}_{B(T)}$ coincide with the cones of $\mathcal{F}_{\mathbb{Q}}(T)$. Our main results are the following theorems.

Theorem 1.1 The 4-punctured sphere has the Null Tangle Property.

Theorem 1.2 Let $B$ be the exchange matrix shown in Figure 1 arising from a triangulation of the 4punctured sphere. Then a complete list of universal geometric coefficients for $B$ is obtained as follows: For each standard form $b / a$ of a rational slope with $0<\frac{b}{a} \leq \infty$, take each vector listed below and apply coordinate permutations indicated by the given permutation group.

$$
\begin{array}{lll}
\text { 1. }\left[-\left\lfloor\frac{b-1}{2}\right\rfloor,\left\lfloor\frac{a}{2}\right\rfloor+1,\left\lfloor\frac{b-a-1}{2}\right\rfloor,-\left\lfloor\frac{b}{2}\right\rfloor,\left\lfloor\frac{a+1}{2}\right\rfloor,\left\lfloor\frac{b-a}{2}\right\rfloor\right] & \langle(14)(25),(25)(36),(123)(456)\rangle \\
\text { 2. }\left[-\left\lfloor\frac{b}{2}\right\rfloor-1,\left\lfloor\frac{a-1}{2}\right\rfloor,\left\lfloor\frac{b-a}{2}\right\rfloor+1,-\left\lfloor\frac{b+1}{2}\right\rfloor,\left\lfloor\frac{a}{2}\right\rfloor,\left\lfloor\frac{b-a+1}{2}\right\rfloor\right] & \langle(14)(25),(25)(36),(123)(456)\rangle
\end{array}
$$




$$
\begin{array}{lll}
\text { 3. }\left[-\left\lfloor\frac{b+1}{2}\right\rfloor,\left\lfloor\frac{a}{2}\right\rfloor,\left\lfloor\frac{b-a+1}{2}\right\rfloor,-\left\lfloor\frac{b}{2}\right\rfloor,\left\lfloor\frac{a+1}{2}\right\rfloor,\left\lfloor\frac{b-a}{2}\right\rfloor\right] & \langle(14),(25),(36),(123)(456)\rangle \\
\text { 4. }[-b, a, b-a,-b, a, b-a] & & \langle(123)(456)\rangle
\end{array}
$$

To prove Theorem 1.2 we classify tagged arcs and describe pairwise compatibility of tagged arcs, thus obtaining a characterization of all tagged triangulations of the 4-punctured sphere. The tagged arcs correspond bijectively to non-closed allowable curves, so we use the classification of tagged arcs to write down a classification of non-closed allowable curves and describe compatibility of non-closed allowable curves. We then separately classify closed allowable curves and extend the description of compatibility. Much of this curve classification is known. (See for example [5, 6].) Finally, we determine the shear coordinates of allowable curves. Having explicit shear coordinates allows us to prove the Null Tangle Property (Theorem 1.1). That property implies that our explicit shear coordinates are universal coefficients, and we obtain Theorem 1.2

Explicit shear coordinates and the description of compatibility among allowable curves lead to an explicit description of the rational part of the mutation fan for the 4-punctured sphere. (This is a fan in $\mathbb{R}^{6}$.) The g-vector fan for the associated cluster algebra appears explicitly as a subfan of the mutation fan, so we also obtain the following theorem.

Theorem 1.3 Let $B$ be the matrix of Theorem 1.2. Then the g-vectors of cluster variables associated to $B^{T}$ are the vectors listed as 1,2, and 3 in Theorem 1.2. with the permutations of entries indicated.

Description of the rational part of the mutation fan leaves open the question of what additional (irrational) cones exist in the mutation fan. We conjecture that these irrational cones are all rays, and all are located on one 2-dimensional plane. Assuming the conjecture, we construct explicit universal coefficients over $\mathbb{R}$ as well.

The results discussed in this introduction allow us to work exclusively with surfaces, tagged arcs and allowable curves, leaving the detailed definitions of cluster algebras of geometric type, universal geometric coefficients, mutation-linear algebra, mutation fans, etc. to [7] and the details of the connection between these concepts and surfaces to [8].

The remainder of this extended abstract is devoted to three tasks. In Section 2 , we describe, in general terms, how key topological facts about the 4-punctured sphere, namely its relationship to the 4-punctured torus and to the $\mathbb{Z}^{2}$-punctured plane, are used in this paper. In Section 3, we give the definitions and results required to understand universal coefficients and the mutation fan for the 4-punctured sphere. In Section 4. we outline the proof of the main theorems and fill in some details.

\section{Sphere, torus, and plane}

In this section, we write $\mathbb{S}-4$ for the 4 -punctured sphere. The main tools in our arguments are two well-known covering spaces of $\mathbb{S}-4$. First, the $\mathbb{S}-4$ is doubly covered by the 4 -punctured torus $\mathbb{T}-4$. One way to picture the covering space is to skewer a donut so that the surface of the donut is punctured in 4 places and so that rotating the skewer through a half turn is a symmetry of the donut. The quotient of this 4-punctured torus modulo this rotation is a 4 -punctured sphere. Second, the $\mathbb{Z}^{2}$-punctured plane $\mathbb{R}^{2}-\mathbb{Z}^{2}$ is a covering space of $\mathbb{S}-4$.

The covering of $\mathbb{S}-4$ by $\mathbb{R}^{2}-\mathbb{Z}^{2}$ has the following description, which also relates to the covering of $\mathbb{S}-4$ by $\mathbb{T}-4$. Consider the group $G$ of rigid motions on $\mathbb{R}^{2}$ generated by half turns about integer points. This group consists of all such rotations together with all even-integer translations (i.e. translations taking 
$(0,0)$ to points with even-integer coordinates). The even-integer translations constitute a normal subgroup $H$ of $G$ of index 2 . The quotient $\left(\mathbb{R}^{2}-\mathbb{Z}^{2}\right) / H$ is $\mathbb{T}-4$ and the quotient $\left(\mathbb{R}^{2}-\mathbb{Z}^{2}\right) / G$ is $\mathbb{S}-4$. In each case, the natural map to the quotient is a covering map. The 2-element quotient group $G / H$ acts on $\mathbb{T}-4$ and $(\mathbb{T}-4) /(G / H)$ is $\mathbb{S}-4$, so the covering map from $\left(\mathbb{R}^{2}-\mathbb{Z}^{2}\right) / G$ to $\mathbb{S}-4$ factors as the composition of the covering map from $\mathbb{R}^{2}-\mathbb{Z}^{2}$ to $\mathbb{T}-4$ followed by the covering map from $\mathbb{T}-4$ to $\mathbb{S}-4$.

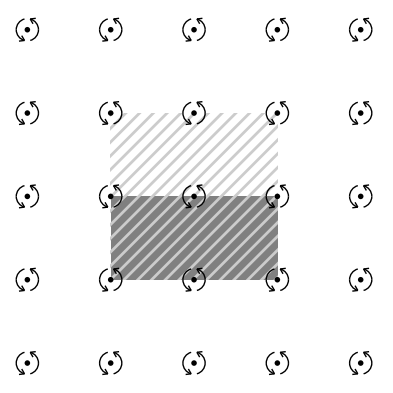

Fig. 2: Fundamental domains for $G$ and $H$ on $\mathbb{R}^{2}-\mathbb{Z}^{2}$

These constructions are pictured in Figures 2, 3, and 4, Figure 2 shows $\mathbb{R}^{2}$ with the points $\mathbb{Z}^{2}$ marked by dots and an indication of half-turns. A fundamental domain for the action of $G$ is indicated by a dark gray rectangle, and a fundamental domain for $H$ is indicated by the entire striped square (including the dark gray rectangle). Figure 3 depicts $\left(\mathbb{R}^{2}-\mathbb{Z}^{2}\right) / H$ as the striped square with edge identifications marked with arrows. The points marked by dots are deleted and, after the edge identifications, these points become the four punctures of the torus. As indicated in the picture, the unique element of $G / H$ acts on $\left(\mathbb{R}^{2}-\mathbb{Z}^{2}\right) / H$ by a half-turn of the square. Figure 4 depicts $\left(\mathbb{R}^{2}-\mathbb{Z}^{2}\right) / G$ as the gray rectangle with edge identifications marked with arrows and deleted points, which become the punctures, labeled as 00, 01, 11, and 10.

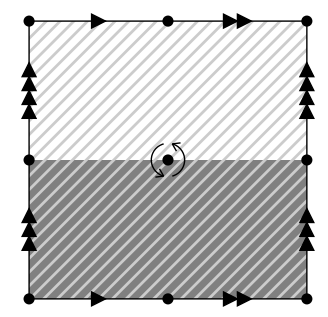

Fig. 3: $\left(\mathbb{R}^{2}-\mathbb{Z}^{2}\right) / H$ and a fundamental domain for $G / H$

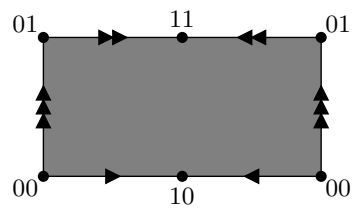

Fig. 4: $\left(\mathbb{R}^{2}-\mathbb{Z}^{2}\right) / G$

As discussed in Section 1 , one main task in constructing the mutation fan/universal coefficients for $\mathbb{S}-4$ is to classify and parametrize the isotopy types of curves in $\mathbb{S}-4$. Before making a precise definition of the curves in question and a precise statement of the classification results, we describe these covering spaces and explain two basic ideas that allow us to use these covering spaces to classify curves. The covering of $\mathbb{T}-4$ by $\mathbb{R}^{2}-\mathbb{Z}^{2}$ essentially agrees ("filling in" the non-even punctures in $\mathbb{R}^{2}-\mathbb{Z}^{2}$ ) with a covering of $\mathbb{T}-1$ by $\mathbb{R}^{2}-(2 \mathbb{Z})^{2}$. The latter covering leads to a classification of curves in $\mathbb{T}-1$ : The curves are parametrized by rational slopes. The covering of $\mathbb{S}-4$ by $\mathbb{T}-4$ allows us to borrow the 
$\mathbb{T}-1$ curve-classification results and obtain curve-classification results for $\mathbb{S}-4$ : Curves are classified by rational slopes and by which, if any, punctures the curve connects.

First, if two curves are isotopic in $\mathbb{S}-4$, then the isotopy lifts to an isotopy between lifts of the curves in $\mathbb{T}-4$. This is also an isotopy between the lifts in $\mathbb{T}-1$. Thus non-isotopic curves in $\mathbb{T}-1$, each missing the other three punctures in $\mathbb{T}-4$, map to non-isotopic curves in $\mathbb{S}-4$.

Second, we can use the covering to establish constraints on the curves that can appear in $\mathbb{S}-4$. Specifically, each curve in $\mathbb{S}-4$ lifts to two curves, related by the rotation of the torus described above, that don't intersect each other. This places constraints on which curves in the torus can appear as lifts of curves in $\mathbb{S}-4$, and thus which curves can appear in $\mathbb{S}-4$.

The connection between sphere and torus also appears in several other places in the proofs of our main results, although these details are omitted from this extended abstract.

It turns out that the argument used in [9] to prove the Null Tangle Property for the once-punctured torus can be adapted to prove the property for the 4-punctured sphere. We also find an interesting connection between the mutation fans for the once-punctured torus and the 4-punctured sphere: There is a 3 -dimensional subspace $U$ of $\mathbb{R}^{6}$ such that the fan induced on $U$ by the mutation fan for the sphere coincides with the mutation fan for the torus.

\section{Surfaces, arcs, and curves}

In this section, we review the basic definitions from [2, 3, 8] of marked surfaces, arcs, triangulations, allowable curves, and so forth, as well as the results linking these constructions to the mutation fan and to universal coefficients. In this paper we are only concerned with two marked surfaces-the once-punctured torus and the 4-times punctured sphere-and we quote the basic definitions and results as they apply to those two surfaces. The reader interested in other marked surfaces should consult the original definitions.

In general, a marked surface is a pair $(\mathbf{S}, \mathbf{M})$ where $\mathbf{S}$ is an orientable surface and $\mathbf{M}$ is a set of points in $\mathbf{S}$ called marked points. The pair $(\mathbf{S}, \mathbf{M})$ must satisfy certain conditions which we do not review here. In this paper, $\mathbf{S}$ is the sphere and $\mathbf{M}$ is a set of four points in the sphere. We will also sometimes consider the torus with exactly one marked point, but reserve the notation $(\mathbf{S}, \mathbf{M})$ for the sphere with four marked points. The constructions we consider are essentially concerned with the topology of the complement $\mathbf{S} \backslash \mathbf{M}$, so we call the marked points punctures and refer to $(\mathbf{S}, \mathbf{M})$ as the 4-punctured sphere.

Definition 3.1 An arc in $(\mathbf{S}, \mathbf{M})$ is a curve $\gamma$ in $\mathbf{S}$ such that:

- The endpoints of $\gamma$ lie in the set $\mathbf{M}$.

- $\gamma$ is non-self-intersecting, except possibly at coinciding endpoints. In the case when its endpoints coincide, we call $\gamma$ a loop.

- $\gamma$ has no punctures in its interior: i.e., $\gamma$ intersects $\mathbf{M}$ at most at its endpoints.

- $\gamma$ is not isotopic to the identity.

We consider arcs up to isotopy relative to $\mathrm{M}$. Two arcs are compatible if there is an isotopy representative of each such that the two representatives do not intersect, except possibly at their endpoints.

Definition 3.2 A tagged arc in (S, $\mathbf{M})$ is an arc $\gamma$ in $(\mathbf{S}, \mathbf{M})$, together with a designation (or "tagging") of each end of the arc as either plain or notched. A tagged arc must also satisfy one additional condition beyond the conditions on an arc:

- $\gamma$ does not bound a disk containing exactly one puncture.

Two tagged arcs are compatible if one of the following conditions holds: 
- The two underlying untagged arcs are the same, and their tagging agrees on one endpoint but not the other.

- The two underlying untagged arcs are distinct and compatible, and any shared endpoints have the same tagging.

A tagged triangulation of $(\mathbf{S}, \mathbf{M})$ is a maximal collection of distinct pairwise compatible tagged arcs.

The most basic reason to consider marked surfaces in connection with cluster algebras is that the combinatorial structure of triangulations of marked surfaces is encoded by an exchange matrix. A deeper reason is that passing from one tagged triangulation to another by "flips" of tagged arcs corresponds to matrix mutation. See [2, Lemma 9.7] for details.

Definition 3.3 Suppose $T$ is a tagged triangulation of $(\mathbf{S}, \mathbf{M})$. The signed adjacency matrix $B(T)$ encodes the structure of $T$. The rows and columns of $B(T)$ are indexed by the arcs in $T$. Due to space restrictions, here we define $B(T)$ only in the case where all of the tagged arcs of $T$ are tagged plain everywhere. In this case, $T$ defines a decomposition of $\mathbf{S}$ into triangles, with each arc shared by two different triangles. For each pair $\alpha, \beta$ of arcs, the $\alpha \beta$-entry of $B(T)$ is the sum of contributions from each triangle of $T$. A triangle contributes +1 if $\alpha$ and $\beta$ are arcs of the triangle with $\alpha$ immediately preceding $\beta$ in a clockwise traversal of the triangle, or contributes -1 if the same is true for a counterclockwise traversal. The contribution is 0 otherwise. An example of this definition appears in Figure 1

Aside from arcs, it is useful to consider an additional class of curves in $(\mathbf{S}, \mathbf{M})$.

Definition 3.4 An allowable curve in $(\mathbf{S}, \mathbf{M})$ is a non-self-intersecting curve in $\mathbf{S} \backslash \mathbf{M}$, not homotopic to a constant curve, that either

- spirals into a puncture at both ends, or

- is closed.

We consider allowable curves up to isotopy. A curve may spiral into a puncture in a clockwise or counterclockwise manner, and curves that spiral in opposite directions are considered to be different curves. Two allowable curves are compatible if either there is an isotopy representative of each such that the two representatives do not intersect, or the two curves coincide except that their spiral direction disagrees at exactly one end. An (integral) quasi-lamination is a collection of pairwise compatible allowable curves with positive integer weights.

Given a tagged arc $\alpha$ in $(\mathbf{S}, \mathbf{M})$ there is a unique curve $\kappa(\alpha)$ which coincides with $\alpha$ except that, at each endpoint $p$, if $\alpha$ is tagged plain at $p$, then $\kappa(\alpha)$ spirals clockwise into $p$, and if $\alpha$ is tagged notched at $p$, then $\kappa(\alpha)$ spirals counterclockwise into $p$. It is immediate from the definitions that $\kappa$ is a bijection from tagged arcs to allowable curves that are not closed, and that two tagged arcs $\alpha$ and $\gamma$ are compatible if and only if the allowable curves $\kappa(\alpha)$ and $\kappa(\gamma)$ are compatible. (See also [2, Definition 17.2] and [8, Lemma 5.1].) Thus $\kappa$ takes tagged triangulations to maximal collections of non-closed pairwise compatible allowable curves. Despite this partial correspondence via the map $\kappa$, it is useful to consider allowable curves and quasi-laminations separately from tagged arcs and tagged triangulations in order to define shear coordinates.

Definition 3.5 (Shear coordinates) Given a lamination $L$ and a tagged triangulation $T$, the shear coordinate vector is a vector $\mathbf{b}(T, L)=\left(b_{\gamma}(T, L): \gamma \in T\right)$ indexed by the tagged arcs $\gamma$ in $T$. We define shear coordinates here only in the case where all of the tagged arcs of $T$ are tagged plain everywhere. 
The shear coordinates record how a lamination interacts with each arc and the two triangles that share it. Each allowable curve $\lambda \in L$ contributes a quantity $b_{\gamma}(T, \lambda)$, and $b_{\gamma}(T, L)$ is the sum $\sum_{\lambda \in L} w_{\lambda} b_{\gamma}(T, \lambda)$, where $w_{\lambda}$ is the weight of $\lambda$ in $L$. To compute $b_{\gamma}(T, \lambda)$, we apply, if necessary, an isotopy to $\lambda$ so as to minimize the number of intersection points of $\lambda$ with $\gamma$. Then $b_{\gamma}(T, \lambda)$ is the sum, over each intersection of $\lambda$ with $\gamma$, of a number that is $-1,0$, or 1 . The number is \pm 1 as shown in Figure 5 if $\lambda$ intersects the two triangles as shown in the figure, and 0 otherwise. The arc $\gamma$ is the diagonal of the square pictured and the curve $\lambda$ is the vertical or horizonal dashed line.
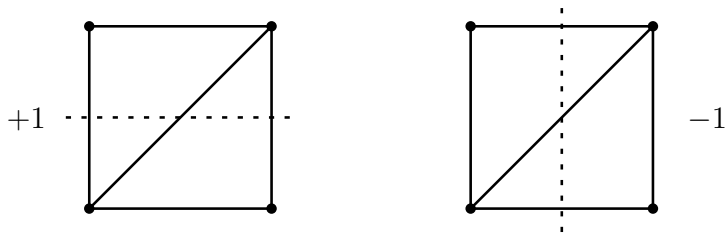

Fig. 5: Shear coordinates

The key property of a marked surface that enables results about the mutation fan and about universal coefficients is the Null Tangle Property.

Definition 3.6 A tangle in (S, M) is an arbitrary finite collection $\Xi$ of allowable curves, each with an integer weight. In contrast with the definition of a lamination, there is no requirement of pairwise compatibility of curves and no requirement of positivity of weights. The shear coordinates $\mathbf{b}(T, \Xi)$ of a tangle $\Xi$ with respect to a tagged triangulation $T$ are computed as the weighted sum of the shear coordinates, with respect to $T$, of the curves in the tangle. A tangle is trivial if all of the weights are zero. A null tangle is a tangle $\Xi$ such that, for any tagged triangulation $T$, the shear coordinates $\mathbf{b}(T, \Xi)$ are zero. The Null Tangle Property is the property of a marked surface that if $\Xi$ is a null tangle then it is trivial.

The Null Tangle Property was proved in [8] for a family of surfaces coincidentally equal to the surfaces of finite growth (see [2, Section 11]), and for the once-punctured torus in [9]. We prove it in this work for the 4-punctured sphere (Theorem 1.1].

Definition 3.7 Fix a tagged triangulation $T$. For any collection $\Lambda$ of pairwise compatible allowable curves, let $C_{\Lambda}$ be the nonnegative $\mathbb{R}$-linear span of the shear coordinates $\{\mathbf{b}(T, \lambda): \lambda \in \Lambda\}$. Let $\mathcal{F}_{\mathbb{Q}}(T)$ be the collection of all cones $C_{\Lambda}$. It is shown in [8. Theorem 4.10] that $\mathcal{F}_{\mathbb{Q}}(T)$ is a rational simplicial fan. We call $\mathcal{F}_{\mathbb{Q}}(T)$ the rational quasi-lamination fan for $T$.

We can now quote the results of [8] that we need. The following theorem is the combination of parts of [8, Theorem 4.10], [7, Theorem 8.7], [2, Proposition 7.10], [8, Theorem 7.3], and [7, Theorem 4.4].

Theorem 3.8 Suppose (S, M) has the Null Tangle Property and let $T$ be any tagged triangulation of $(\mathbf{S}, \mathbf{M})$. Then

- The fan $\mathcal{F}_{\mathbb{Q}}(T)$ is the rational part of the mutation fan $\mathcal{F}_{B(T)}$.

- The $\mathbf{g}$-vector fan of $B(T)$ is the set of all cones $C_{\Lambda}$ such that $\Lambda$ is a collection of pairwise compatible non-closed allowable curves. (This statement must be modified if $(\mathbf{S}, \mathbf{M})$ is a closed surface with exactly one puncture.)

- The shear coordinates of allowable curves constitute universal geometric coefficients for $B(T)$. 


\section{Proof outline, and some details}

In this section, we outline the proofs of the main theorems and give some details. We continue to let $(\mathbf{S}, \mathbf{M})$ denote the 4-punctured sphere. We rely heavily on the covering maps described in Section 2. Each of these covering maps extends continuously to a map on the corresponding unpunctured spaces, taking punctures to punctures. We will thus speak of the action of the covering maps on punctures, even though the maps on the unpunctured spaces are only branched coverings.

We index the four punctures in the sphere according to their pre-images in the plane. Each integer point in $\mathbb{R}^{2}$ maps to a puncture of the sphere, and two points map to the same puncture if and only if their coordinates agree modulo 2 . Accordingly, we index the punctures as $v_{p q}$ (or when necessary $v_{p, q}$ ) where $p$ and $q$ are integers mod two. This indexing of the punctures is consistent with the numbering in Figure 4

Each arc $\gamma$ in $(\mathbf{S}, \mathbf{M})$ is in particular the image of some continuous map from the closed unit interval to $\mathbf{S}$, with endpoints of the interval mapping into $\mathbf{M}$. The definition ensures that the restriction $\left.\gamma\right|_{\mathbf{S} \backslash \mathbf{M}}$ of $\gamma$ to the open unit interval is a curve in $\mathbf{S} \backslash \mathbf{M}$. The endpoints of $\gamma$ are recovered as the limits (within $\mathbf{S}$ ) of $\left.\gamma\right|_{\mathbf{S} \backslash \mathbf{M}}$. When we consider the covering of $\mathbf{S} \backslash \mathbf{M}$ by the 4-punctured torus or by the $\mathbb{Z}^{2}$-punctured plane, we must consider $\left.\gamma\right|_{\mathbf{S} \backslash \mathbf{M}}$. However, any lift of $\left.\gamma\right|_{\mathbf{S} \backslash \mathbf{M}}$ to one of the covering spaces is a curve limiting, at each end, to a puncture. In particular, any lifting of $\left.\gamma\right|_{\mathbf{S} \backslash \mathbf{M}}$ is the restriction to the open interval of a unique curve $\tilde{\gamma}$ whose endpoints are punctures of the covering space. Thus it makes sense to talk about lifting an arc $\gamma$ to one of the covering spaces, rather than lifting only its restriction $\left.\gamma\right|_{\mathbf{S} \backslash \mathbf{M}}$. (Some care must be taken when trying to apply "unique lifting" results to arcs.) Similarly, it makes sense to speak of projecting arcs down from the covering spaces.

The first step in proving our main results is to classify allowable curves and describe their compatibility. Because it is no harder, and because tagged arcs are of independent interest, we classify the non-closed allowable curves by first classifying tagged arcs and then applying the map $\kappa$.

\subsection{Tagged arcs and tagged triangulations}

Tagged arcs are specified by, among other data, a rational slope, i.e. a rational number or $\infty$. Rational slopes are in one-to-one correspondence with formal expressions $b / a$ with $\operatorname{gcd}(a, b)=1$ such that $a \geq 0$ and such that $b=1$ whenever $a=0$. Such an expression is the standard form of a rational slope. Consider an integer point $[p, q] \in \mathbb{Z}^{2}$ and the standard form $b / a$ of a rational slope. Because $b / a$ is in standard form, the straight line segment connecting $[p, q]$ to $[p+a, q+b]$ intersects no other integer points. Let $\operatorname{arc}\left(a, b,\left\{v_{p, q}, v_{p+a, q+b}\right\}\right)$ be the projection to $(\mathbf{S}, \mathbf{M})$ of the line segment connecting $[p, q]$ to $[p+a, q+b]$. This is an arc in $(\mathbf{S}, \mathbf{M})$ connecting the punctures $v_{p, q}$ and $v_{p+a, q+b}$. Since the indices on punctures are interpreted mod 2, this arc only depends on $[p, q]$ modulo 2 . The set braces in the notation remind us that $v_{p, q}$ and $v_{p+a, q+b}$ appear symmetrically in the construction, because the line segment connecting $[p+a, q+b]$ to $[p+2 a, q+2 b]$ projects to the same arc in $(\mathbf{S}, \mathbf{M})$ as the line segment connecting $[p, q]$ to $[p+a, q+b]$. Since $b / a$ is in standard form, $b$ and $a$ are not both multiples of 2 , so the set $\left\{v_{p, q}, v_{p+a, q+b}\right\}$ has two distinct elements. For any standard form $b / a$, there are exactly two choices for the set $\left\{v_{p, q}, v_{p+a, q+b}\right\}$.

We use a similar notation for tagged arcs in $(\mathbf{S}, \mathbf{M})$ by attaching a superscript $v_{p, q}$ and/or $v_{p+a, q+b}$ to indicate notched tagging of the arc at that endpoint. There are four tagged arcs whose underlying $\operatorname{arc}$ is $\operatorname{arc}\left(a, b,\left\{v_{p, q}, v_{r, s}\right\}\right)$. They $\operatorname{are} \operatorname{arc}\left(a, b,\left\{v_{p, q}, v_{r, s}\right\}\right), \operatorname{arc}\left(a, b,\left\{v_{p, q}^{\boldsymbol{q}}, v_{r, s}\right\}\right)$, $\operatorname{arc}\left(a, b,\left\{v_{p, q}, v_{r, s}^{\bullet}\right\}\right)$, and $\operatorname{arc}\left(a, b,\left\{v_{p, q}, v_{r, s}^{\bullet}\right\}\right)$. Let $A$ be the set of triples $(a, b, E)$ such that $b / a$ is 

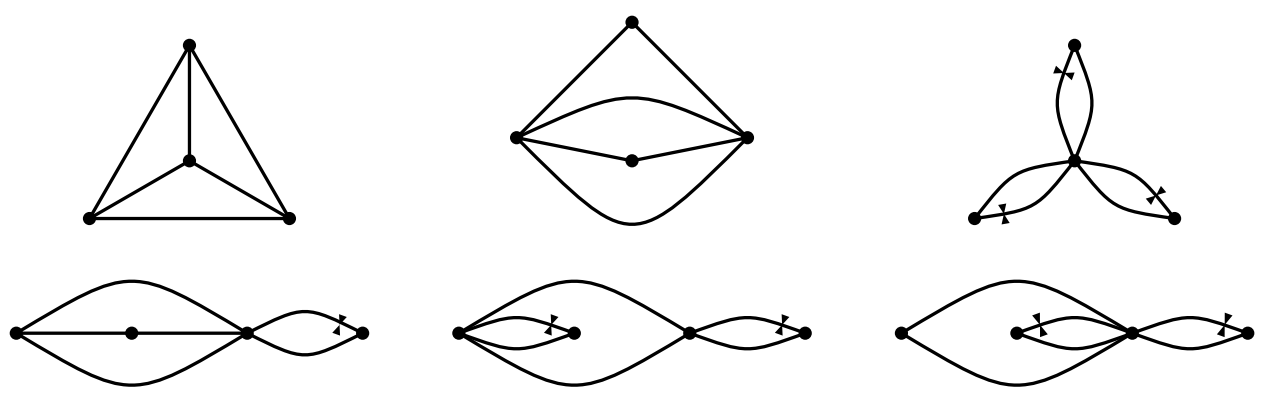

Fig. 6: tagged triangulations of the 4-punctured sphere

the standard form for a rational slope and $E$ is $\left\{v_{p, q}, v_{r, s}\right\},\left\{v_{p, q}^{\bullet \backslash}, v_{r, s}\right\},\left\{v_{p, q}, v_{r, s}^{\bullet \mathbf{A}}\right\}$, or $\left\{v_{p, q}^{\bullet \mathbf{4}}, v_{r, s}^{\bullet \mathbf{4}}\right\}$ with $[p, q]+[a, b]=[r, s]$ modulo 2 .

Proposition 4.1 The map $(a, b, E) \mapsto \operatorname{arc}(a, b, E)$ is a bijection from $A$ to $\{$ tagged arcs in $(\mathbf{S}, \mathbf{M})\}$.

In particular, there are no tagged loops in $(\mathbf{S}, \mathbf{M})$. This is easily understood: a tagged loop separates the sphere into two disks, one of which contains 0 or 1 punctures, but both possibilities are specifically excluded for tagged arcs in Definition 3.2

Proposition 4.2 Two tagged arcs whose underlying arcs coincide are compatible if and only if their tagging agrees at exactly one of their endpoints. Two tagged $\operatorname{arcs} \operatorname{arc}(a, b, E)$ and $\operatorname{arc}(c, d, F)$ whose underlying arcs do not coincide are compatible if and only if their taggings agree at each shared endpoint and $|a d-b c|$ equals the number of shared endpoints $(0,1$, or 2$)$.

We call two distinct tagged arcs a coinciding pair if their underlying arcs coincide and their taggings make them compatible as described in Proposition 4.2 Rational slopes $b / a$ and $d / c$ with $|a d-b c|=1$ are sometimes called Farey pairs. Accordingly, we call non-coinciding compatible pairs $\operatorname{arc}(a, b, E)$ and $\operatorname{arc}(c, d, F)$ Farey-0 pairs (or parallel pairs), Farey-1 pairs, or Farey-2 pairs according to their number of shared endpoints. We overload the terms by also referring to "Farey-1 (or Farey-2) pairs of slopes."

With a description of compatibility of tagged arcs in hand, we describe the tagged triangulations of $(\mathbf{S}, \mathbf{M})$. By [2, Theorem 7.9], each tagged triangulation has the same number of arcs, and we have seen in Figure 1 that this number is 6 . We illustrate the six combinatorial types of tagged triangulations in Figure 6. These pictures are drawn, not in the sphere illustrated in Figure 4, but rather in a more conventional sphere: the plane of the page together with the "point at infinity." Each combinatorial type can be tagged in more than one way. Specifically, at each puncture, except the punctures where there are already two distinct tags, endpoints of arcs can all be tagged plain or all be tagged notched, and this choice can be made independently at each puncture. To arrive at this list of combinatorial types, we view a tagged triangulation as a graph in which the punctures are vertices and arcs are edges. We first determine the possible degree sequences the corresponding graph may have. To differentiate between tagged triangulations with the same degree sequence, but different combinatorial types (eg. the upper right and lower right tagged triangulations in Figure 6) we also specify the number of coinciding pairs of arcs in the triangulation.

To completely understand tagged triangulations, we need to determine, for each combinatorial type, precisely what additional data are required to specify a unique tagged triangulation. In this extended 
abstract, we do this only for two of the combinatorial types.

We begin with the triangulation with degree sequence $3,3,3,3$, which we call the tetrahedron, and which appears as the upper left picture in Figure 6. Note that a Farey-2 pair divides the sphere into two once-punctured monogons, and the puncture inside each monogon must have degree 2. Any coinciding pair must be incident to a puncture of degree 2. Since no puncture has degree 2 in the tetrahedron, the tetrahedron contains no coinciding or Farey-2 pairs. It follows that the tagging at each puncture can be chosen arbitrarily, subject to the restriction that all taggings agree at each puncture. It also follows that any pair of tagged arcs incident to a single puncture form a Farey-1 pair and any pair of tagged arcs sharing no punctures form a Farey-0 pair. Thus once the tagging at each puncture has been chosen, selecting any three arcs which form a Farey-1 triple (a triple, each pair of which is a Farey-1 pair) determines the remaining three arcs of the tagged triangulation. We conclude that there is exactly one tetrahedron for each choice of taggings and each Farey-1 triple.

Now consider a tagged triangulation with degree sequence $6,2,2,2$ containing three coinciding pairs, as shown in the upper right of Figure 6. We will call such a triangulation a pinwheel. After we select a vertex to have degree 6 and choose a tagging for that vertex, each pair of coinciding arcs is uniquely determined by a choice of slope. Since any two non-coinciding arcs in the pinwheel share exactly one vertex, the three slopes form a Farey-1 triple. Therefore the pinwheel triangulations are specified by a choice of vertex, a tagging of the vertex, and a Farey-1 triple.

\subsection{Allowable curves and the rational lamination fan}

Since $\kappa$ is a bijection between tagged triangulations and maximal pairwise compatible sets of non-closed allowable curves, Proposition 4.1 can be rephrased as a classification of non-closed allowable curves. The classification of allowable curves is completed by the following proposition, which is well-known. (See for example [5, 6].) We omit the details here.

Proposition 4.3 The allowable closed curves in $(\mathbf{S}, \mathbf{M})$ are the images, under the covering map, of straight lines in $\mathbb{R}^{2} \backslash \mathbb{Z}^{2}$ with (possibly infinite) rational slope. Two such images are isotopic if and only if the corresponding lines have the same slope.

Similarly, the classification of tagged triangulations can be rephrased as a classification of maximal pairwise compatible sets of non-closed allowable curves. These correspond to full-dimensional (6-dimensional) cones in the mutation fan $\mathcal{F}_{B}$. To complete the description of the rational part of the mutation fan, we consider maximal sets of pairwise compatible allowable curves containing at least one closed allowable curve. Any closed allowable curve in $(\mathbf{S}, \mathbf{M})$ cuts $(\mathbf{S}, \mathbf{M})$ into two twice-punctured disks, and no other closed allowable curve can be contained in either twice-punctured disk. Thus no two distinct closed allowable curves can be compatible. It is also not hard to convince oneself that a closed allowable curve and a non-closed allowable curve are compatible if and only if they are specified by the same slope. (In this case, the non-closed allowable curve spirals into the two punctures in one of the twice-punctured disks.) Thus there are exactly 8 allowable curves compatible with a given closed allowable curve.

Any maximal pairwise compatible set of allowable curves contains at most one closed curve. Each given closed allowable curve is contained in exactly 16 maximal collections of pairwise compatible allowable curves, each containing five curves. A given collection is obtained by choosing, for each of the two twice-punctured disks, which of the punctures should have opposite spiral directions and then specifying the spiral direction at the other puncture. These maximal pairwise compatible sets with one closed curve correspond to maximal 5-dimensional cones in $\mathcal{F}_{B}$. 

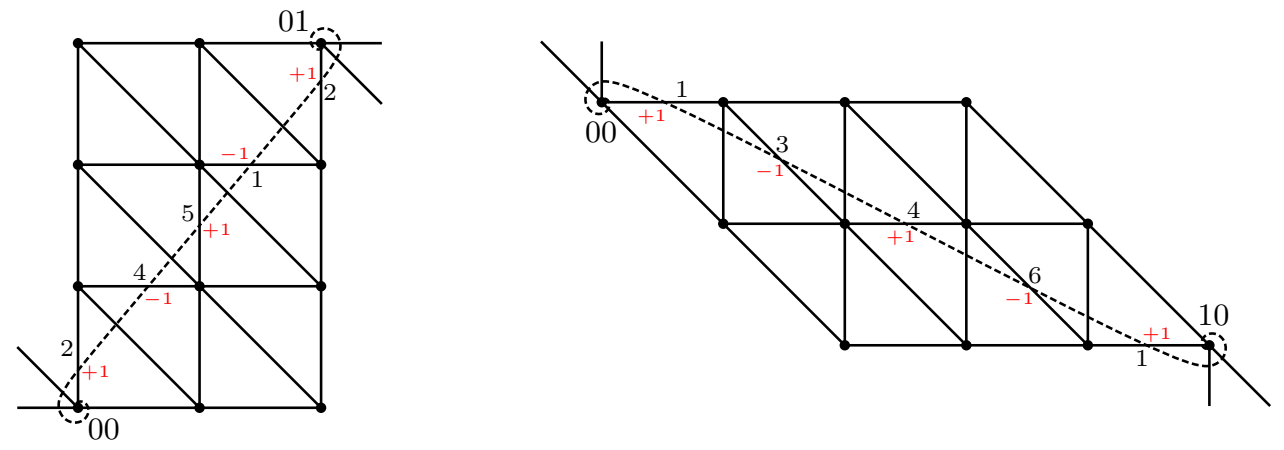

Fig. 7: Some shear coordinate calculations

\subsection{The Null Tangle Property}

With the classification of allowable curves in hand, we can prove the Null Tangle Property. The verification of the Null Tangle Property is a technical argument following the outline of the proof in [9] of the Null Tangle Property for the once-punctured torus. The first piece of the argument is to show that, in any null tangle, the weight of any non-closed curve is zero. Then one verifies that any null tangle involving only closed curves is trivial. We omit the details.

\subsection{Shear coordinates}

As before, let $T=\left\{\gamma_{i}\right\}$ be the triangulation of Figure 1. With the Null Tangle Property established, Theorem 3.8 says that the set of all shear coordinate vectors with respect to $T$ constitutes universal geometric coefficients for $B(T)$. We conclude our outline of the proofs of the main results by describing the computation of shear coordinates that verifies Theorem 1.2

Let $\lambda$ be an allowable curve. As described in Definition 3.5, computing the shear coordinate $b_{\gamma_{i}}(T, \lambda)$ amounts to counting and categorizing the intersections of $\lambda$ with $\gamma_{i}$ into crossings of type $-1,1$ (nontrivial) and 0 (trivial) and summing these contributions. This is most easily done by taking a lift $\bar{\lambda}$ of $\lambda$ to $\mathbb{R}^{2}$ (specifically, the lift to the positive quadrant with one endpoint at the origin) and examining intersections with the lifts of $\gamma_{i}$ there. For example, let $\lambda$ be the projection of the curve $\bar{\lambda}$ in $\mathbb{R}^{2}$ shown in the left picture of Figure 7

Each arc shown in the figure is the lift of an arc of the triangulation $T$ in the 4-punctured sphere, and some of these are labeled with the numbering from Figure 1 Nonzero contributions to shear coordinates are shown in red in the picture, adding up to $[-1,2,0,-1,1,0]$.

Initially, we restrict ourselves to curves with positive slopes and one endpoint at puncture $v_{00}$ (or, in the case of closed curves, "near" $v_{00}$ ). We further simplify computation by initially ignoring endpoint spirals, if they exist, and focusing solely on the linear interior of $\bar{\lambda}$. We call any intersections between $\bar{\lambda}$ and lifts of $\gamma_{i}$ not arising from spirals "interior" intersections. Recalling our construction of allowable curves in Definition 3.4 as $\kappa(\alpha)$ for a tagged arc $\alpha$, this simplification is natural: essentially, interior intersections are those arising from $\alpha^{\prime}$, the arc $\alpha$ with taggings removed. We then add shear coordinate contributions from different types of tagging, (i.e., spiraling) on a case-by-case basis.

The triangulation $T$ was chosen specifically to correspond to the fixed triangulation $T_{0}$ in [9, Section 5], allowing us to group arcs in $T$ into pairs: 'horizontal' arcs $\gamma_{1}$ and $\gamma_{4}$, 'vertical' arcs $\gamma_{2}$ and $\gamma_{5}$, and 
'diagonal' arcs $\gamma_{3}$ and $\gamma_{6}$, corresponding, respectively, to the single $\operatorname{arcs} \gamma_{1}, \gamma_{2}, \gamma_{3} \in T_{0}$. This construction allows us to reuse much of the proof of [9, Proposition 5.1]. It can be shown that interior intersections of $\bar{\lambda}$ with lifts of 'horizontal' (respectively, 'vertical') arcs are always non-trivial and alternate between $\gamma_{1}$ and $\gamma_{4}$ (respectively, $\gamma_{2}$ and $\gamma_{5}$ ). We show further that while not all interior intersections of $\bar{\lambda}$ with diagonal arcs are non-trivial, those that are alternate between $\gamma_{3}$ and $\gamma_{6}$. The formulas in Theorem 1.2 follow after some straightforward computations.

The permutations of entries arise in Theorem 1.2 for two different reasons. First, we restricted to curves starting at $/$ near $v_{00}$. To consider other starting points, we can translate the lift, and this translation gives a permutation of arcs. For example, to change the starting point to $v_{01}$, we translate the lift one unit up, and this acts as the permutation (14)(36) on the indices $i$ of the $\gamma_{i}$. Second, we restricted to positive slopes. Shear coordinates for curves with negative slopes can also be obtained by permutations of coordinates, using the idea that appeared in [9. Section 5]. We omit the details here, but provide an example in the two shear coordinate calculations of Figure 7

\section{Acknowledgements}

The authors thank Dylan Allegretti, Bill Floyd, Vladimir Fock, Alexander Goncharov, Allen Hatcher, and Lee Mosher for helpful responses to email queries.

\section{References}

[1] W. Floyd and A. Hatcher. Curves on the 4-punctured sphere: 31 charts. Unpublished manuscript, 1980. Available at http://www.math.cornell.edu/ hatcher/Papers/31charts/thirtyone.html.

[2] S. Fomin, M. Shapiro and D. Thurston, Cluster algebras and triangulated surfaces. Part I: Cluster complexes. Acta Math. 201 (2008), no. 1, 83-146.

[3] S. Fomin and D. Thurston, Cluster algebras and triangulated surfaces. Part II: Lambda lengths. Preprint, 2012. (arXiv:1210.5569)

[4] S. Fomin and A. Zelevinsky, Cluster algebras IV: Coefficients. Compos. Math. 143 (2007), no. 1, $112-164$.

[5] L. Keen and C. Series, The Riley slice of Schottky space. Proc. London Math. Soc. (3) 69 (1994), no. $1,72-90$.

[6] Y. Komori and C. Series, The Riley slice revisited. The Epstein birthday schrift, 303-316. Geom. Topol. Monogr., 1, Geom. Topol. Publ., Coventry, 1998.

[7] N. Reading, Universal geometric cluster algebras. Math. Z. 277 (2014), no. 1-2, 499-547.

[8] N. Reading, Universal geometric cluster algebras from surfaces. Trans. Amer. Math. Soc. 366 (2014), no. $12,6647-6685$.

[9] N. Reading, Universal geometric coefficients for the once-punctured torus. Preprint, 2012. (arXiv:1212.1351) 